\title{
Farmacocinetica del calcidiolo e del colecalciferolo dopo somministrazione orale in individui sani con differente BMI e in adulti con malassorbimento intestinale
}

\author{
Salvatore Minisola ${ }^{1}$
}

Accettato: 24 luglio 2021 / Pubblicato online: 9 novembre 2021

(c) The Author(s), under exclusive licence to Springer Nature Switzerland AG 2021

Commento a:

A pilot-randomized, double-blind crossover trial to evaluate the pharmacokinetics of orally administered 25-hydroxyvitamin D3 and vitamin D3 in healthy adults with differing BMI and in adults with intestinal malabsorption.

N. Charoenngam, T.A. Kalajian, A. Shirvani, G.H Yoon, S. Desai, A. McCarthy, C.M. Apovian, M.F. Holick. Am J Clin Nutr (2021) 114(3):1189-1199

Vi è un continuo dibattito sull'utilità della supplementazione con vitamina $\mathrm{D}$ sia in condizioni fisiologiche sia, soprattutto, nella prevenzione di alcune malattie. Recentemente, tale dibattito si è ulteriormente ravvivato dalla prospettata utilità della vitamina D nel prevenire l'infezione da COVID-19 o nel modularne positivamente il decorso. All'interno di questo dibattito, un altro motivo di discussione è costituito dalla forma di vitamina $\mathrm{D}$ da utilizzare.

Le tre forme principali di vitamina $\mathrm{D}$ sono infatti rappresentate da: colecalciferolo (vitamina $\mathrm{D}_{3}$ ), calcidiolo $\left[25(\mathrm{OH}) \mathrm{D}_{3}\right]$ e $1,25(\mathrm{OH})_{2} \mathrm{D}_{3}$ (calcitriolo), anche se esistono altri composti utilizzati in commercio ma con minore diffusione (ergocalciferolo, alfacalcidiolo).

Gli autori di questa indagine hanno valutato la farmacocinetica del colecalciferolo e del calcidiolo in soggetti normali e in pazienti con varie forme di malassorbimento intestinale.

Il risultato principale è rappresentato dal rilievo che la biodisponibilità della somministrazione orale di $25(\mathrm{OH}) \mathrm{D}_{3}$ è uguale sia nei soggetti normali, sia nei pazienti con malassorbimento. Ciò dipende molto probabilmente dal fatto che, essendo la $25(\mathrm{OH}) \mathrm{D}_{3}$ più idrofilica, viene direttamente

S. Minisola

salvatore.minisola@uniroma1.it

1 Dipartimento di Scienze Cliniche, Internistiche e

Anestesiologiche, "Sapienza” Università di Roma, Roma, Italia assorbita nel sistema portale e distribuita nella circolazione. Il percorso metabolico della vitamina $\mathrm{D}_{3}$ è invece più complesso, poiché questa deve essere incorporata nei chilomicroni e giungere nel sistema linfatico prima di passare nella circolazione generale. In aggiunta a ciò, la biodisponibilità del calcidiolo è risultata essere maggiore rispetto al colecalciferolo nei soggetti normali obesi.

Anche se questo lavoro ha alcuni limiti, il principale dei quali rappresentato dai ridotti livelli di $25(\mathrm{OH}) \mathrm{D}$ raggiunti dopo somministrazione di calcidiolo rispetto a lavori pubblicati nel passato [1-3], tuttavia rappresenta un contributo importante poiché rafforza il concetto che in almeno due condizioni (cioè il malassorbimento intestinale e l'obesità) si dovrebbe preferire la somministrazione di calcidiolo rispetto a quella di colecalciferolo. Alternativamente, le dosi di quest'ultimo dovrebbero essere aumentate per raggiungere $\mathrm{i}$ valori di sufficienza di vitamina $D$.

\section{Bibliografia}

1. Russo S, Carlucci L, Cipriani C et al (2011) Metabolic changes following $500 \mu \mathrm{g}$ monthly administration of calcidiol: a study in normal females. Calcif Tissue Int 89(3):252-257

2. Bischoff-Ferrari HA, Dawson-Hughes B, Stöcklin E et al (2012) Oral supplementation with $25(\mathrm{OH}) \mathrm{D} 3$ versus vitamin D3: effects on $25(\mathrm{OH}) \mathrm{D}$ levels, lower extremity function, blood pressure, and markers of innate immunity. J Bone Miner Res 27(1):160-169

3. Minisola S, Cianferotti L, Biondi P et al (2017) Correction of vitamin D status by calcidiol: pharmacokinetic profile, safety, and biochemical effects on bone and mineral metabolism of daily and weekly dosage regimens. Osteoporos Int 28(11):3239-3249

Nota della casa editrice Springer Nature rimane neutrale in riguardo alle rivendicazioni giurisdizionali nelle mappe pubblicate e nelle affiliazioni istituzionali. 